\title{
ON THE CONVERGENCE OF NEWTON-LIKE METHODS
}

\author{
IOANNIS K. ARGYROS AND FERENC SZIDAROVSZKY
}

\begin{abstract}
This paper examines conditions for the convergence of generalized Newton-like methods, and estimates the speed of convergence.
\end{abstract}

\section{Introduction}

In this paper we are concerned with the problem of finding conditions for the convergence of generalized Newton-like methods to a common fixed point $x^{*}$ of mappings $f_{k}(k \geq 0)$ defined on a subset of a Banach space $B$. Such a problem is clearly important in numerical analysis since many applied problems reduce to locating fixed points $x^{*}$ of such mappings. For example, iterations of the above type are extremely important in solving optimization problems as well as linear and nonlinear equations. A very important field of such applications can also be found in solving optimization problems in economy and solving nonlinear inputoutput systems (see ex. Fujimoto, [3], La Salle, [5], Okuguchi, [6], Okuguchi \& Szidarovszky, [7], Ortega \& Rheinboldt, [8], Polak, [9], Tishyadihigama, et al, [11]).

In particular, set $U(0, R)=\{x \in B /\|x\| \leq R\}$, consider the Newton-like iterates

$$
x_{k+1}=x_{k}-A_{k}\left(x_{k}\right)^{-1}\left(f_{1 k}\left(x_{k}\right)+f_{2 k}\left(x_{k}\right)\right)
$$

Received September 17, 1990; revised April 15, 1992.

(1980) A.M.S. classification codes: 47D15, 47H17, 65R20, 65J10.

Key words and phrases: Newton-like method, point-to-point mapping, fixed points. 
for approximating a common fixed point $x^{*}=0$ of equations

$$
f_{k}(x)=f_{1 k}(x)+f_{2 k}(x) \quad(k \geq 0)
$$

Here, $f_{k}, f_{1 k}, f_{2 k}$ are mappings defined on $U(0, R)$ with values in $B, A_{k}(\cdot)$ denote linear mappings which approximate the Frechet-derivative $f_{1 k}^{\prime}(x)$ of $f_{1 k}$ at $x \in U(0, R)$. The assumption $x^{*}=0$, involves no loss of generality, since any solution $x^{*}$ can be transformed into 0 by introducing the transformed mapping $q_{k}(x)=f_{k}\left(x+x^{*}\right)-x^{*}(k \geq 0)$.

\section{Convergence Analysis}

We assume that for all $k, k \geq 0$, and each fixed $r \in[0, R]$

(A) $A_{k}(0)^{-1}$ exists and for all $x, y \in U(0, r) \subseteq U(0, R)$,

$$
\begin{aligned}
\left\|A_{k}(0)^{-1}\left(A_{k}(x)-A_{k}(0)\right)\right\| & \leq w_{0}(\|x\|)+b \\
\left\|A_{k}(0)^{-1}\left(f_{1 k}^{\prime}(t x)-A_{k}(x)\right)\right\| & \leq w(t\|x\|)+c, t \in[0,1]
\end{aligned}
$$

and

$$
\left\|A_{k}(0)^{-1}\left(f_{2 k}(x)-f_{2 k}(y)\right)\right\| \leq e(r)\|x-y\|
$$

where $w_{0}, w$ and $e$ are nondecreasing nonnegative functions and constants $b, c$ satisfy $b \geq 0, c \geq 0$ and $b+c<1$. Note that the differentiability of $f_{2 k}$ is not assumed.

The above conditions are more general than the ones considered by Argyros, [1], Dennis, [2], Kantorovich \& Akilov, [4], Ortega \& Rheinboldt, [8], Yamamoto \& Chen, [12], Zabrejko \& Nguen, [13] who treated the above problem when $f_{k}=f, k \geq 0$. They provided sufficient conditions for the convergence of Newton-like iterates (1) to 0 in this special case. We will proceed in a similar manner but for the more general case described above. Our results can be easily reduced to the ones obtained by the above authors when $f_{k}=f, k \geq 0$. However, we will leave that to the motivated reader. 
Define now the functions

$$
x^{*}(r)=\int_{0}^{r} w(s) d s+\int_{0}^{r} e(r) d s+\left(b+c-1+w_{0}(r)\right) r
$$

and

$$
g(r)=1-b-w_{0}(r) \text { for all } r \in[0, R)
$$

Introduce the difference equation

$$
\rho_{k+1}=\rho_{k}+x^{*}\left(\rho_{k}\right) g\left(\rho_{k}\right)^{-1}(k \geq 0), \quad \rho_{o}=R .
$$

We can now formulate the main result:

Theorem. Under Condition $(A)$, assume there exist $x_{0} \in B, R>0$ such that 0 is the unique zero of function $x^{*}(r)$ given by $(6)$ in $[0, R)$. Moreover, suppose $\left\|x_{0}\right\| \leq a \leq R$ and $x^{*}(R) \leq 0$.

Then iterates generated by (1) are well defined for all $k \geq 0$, belong to $U(0, R)$ and converge to 0 with

$$
\left\|x_{k}\right\| \leq \rho_{k} \quad(k \geq 0)
$$

where sequence $\rho_{k}$ which is monotonically decreasing and coverges to 0 is given by (8).

Proof. We will first show that the sequence generated by ( 8 ) is monotonically decreasing and converges to 0 . Since 0 is the unique zero of function $x^{*}(r)$ in $[0, R)$ and $x^{*}(R) \leq 0$,

$$
x^{*}(r)<0 \text { for all } r \in[0, R) .
$$

By using (6) we get

$$
0 \leq \int_{0}^{r} w(s) d s+\int_{0}^{r} e(r) d s<\left(1-b-c-w_{0}(r)\right) r
$$

which implies that

$$
g(r)>0 \text { for all } r \in[0, R)
$$


Using relations (8), (10), (11) and finite induction, it is routine to show that sequence $\rho_{k}$ is monotonically decreasing. Furthermore, iteration (8) can also be written as

$$
\rho_{k+1}=\left[\int_{0}^{\rho_{k}}(w)(s) d s+e\left(\rho_{k}\right) \rho_{k}+c \rho_{k}\right] g\left(\rho_{k}\right)^{-1} \geq 0 \text { for all } k \geq 0
$$

which imply that

$$
0 \leq \rho_{k+1} \leq \rho_{k} \quad(k \geq 0) .
$$

Hence, there exists a $\rho^{*} \in[0, R)$ with $\rho_{k} \rightarrow \rho^{*}$ as $k \rightarrow \infty$. Note that from (8) and the uniqueness of 0 as a zero of $x^{*}(r)$ in $[0, R)$ we conclude that $\rho^{*}=0$.

By induction on $k$ we will show that (9) holds: For $k=0$, (9) becomes $\left\|x_{0}\right\| \leq \rho_{0}=R$, which is true since $a \leq R$ by hypothesis. Assume (9) holds for $k$. From (3) and (11) we get

$$
\left\|A_{k}(0)^{-1}\left(A_{k}\left(x_{k}\right)-A_{k}(0)\right)\right\| \leq w_{0}\left(\rho_{k}\right)+b<1 .
$$

By the Banach lemma on invertible mappings $A_{k}\left(x_{k}\right)$ is invertible. By using identity

$$
A_{k}\left(x_{k}\right)=A_{k}(0)\left[I+A_{k}(0)^{-1}\left(A_{k}\left(x_{k}\right)-A_{k}(0)\right)\right]
$$

we see that

$$
\left\|A_{k}\left(x_{k}\right)^{-1} A_{k}(0)\right\| \leq g\left(\rho_{k}\right)^{-1} \quad(k \geq 0) .
$$

From (1) and the fact that 0 is a fixed point of equation (2) we obtain in turn.

$$
\begin{aligned}
x_{k+1}= & x_{k}-A\left(x_{k}\right)^{-1} f_{k}\left(x_{k}\right) \\
= & -A_{k}\left(x_{k}\right)^{-1}\left[f_{k}\left(x_{k}\right)-A_{k}\left(x_{k}\right) x_{k}\right] \\
= & -A_{k}\left(x_{k}\right)^{-1}\left[f_{1 k}\left(x_{k}\right)+f_{2 k}\left(x_{k}\right)-A_{k}\left(x_{k}\right) x_{k}-\left(f_{1 k}(0)+f_{2 k}(0)\right)\right] \\
= & -A_{k}\left(x_{k}\right)^{-1}\left[\left(f_{1 k}\left(x_{k}\right)-f_{1 k}(0)-A_{k}\left(x_{k}\right) x_{k}\right)+\left(f_{2 k}\left(x_{k}\right)-f_{2 k}(0)\right)\right] \\
= & -A_{k}\left(x_{k}\right)^{-1}\left[\int_{0}^{1} f_{1 k}^{\prime}\left(t x_{k}\right) x_{k} d t-A_{k}\left(x_{k}\right) x_{k}+\left(f_{2 k}\left(x_{k}\right)-f_{2 k}(0)\right)\right] \\
= & -\left[A_{k}\left(x_{k}\right)^{-1} A_{k}(0)\right]\left\{\int _ { 0 } ^ { 1 } A _ { k } ( 0 ) ^ { - 1 } \left[\left(\left(f_{1 k}^{\prime}\left(t x_{k}\right)-A_{k}\left(x_{k}\right)\right) x_{k} d t\right.\right.\right. \\
& \left.\left.+\left(f_{2 k}\left(x_{k}\right)-f_{2 k}(0)\right)\right]\right\} .
\end{aligned}
$$


By taking norms in the above approximation and using the triangle inequality and (13) we obtain

$$
\begin{aligned}
\left\|x_{k+1}\right\|= & \|\left[A_{k}\left(x_{k}\right)^{-1} A_{k}(0)\right]\left\{\int _ { 0 } ^ { 1 } A _ { k } ( 0 ) ^ { - 1 } \left[\left(\left(f_{1 k}^{\prime}\left(t x_{k}\right)-A_{k}\left(x_{k}\right)\right) x_{k}\right) d t\right.\right. \\
& \left.\left.+\left(f_{2 k}\left(x_{k}\right)-f_{2 k}(0)\right)\right]\right\} \| \\
\leq & \left\|A_{k}\left(x_{k}\right)^{-1} A_{k}(0)\right\|\left\{\left\|\int_{0}^{1} A_{k}(0)^{-1}\left(\left(f_{1 k}^{\prime}\left(t x_{k}\right)-A_{k}\left(x_{k}\right)\right) x_{k}\right) d t\right\|\right. \\
& \left.+\left\|A_{k}(0)^{-1}\left(f_{2 k}\left(x_{k}\right)-f_{2 k}(0)\right)\right\|\right\} \\
\leq & \left(\int\left[w\left(t\left\|x_{k}\right\|\right)+c+e\left(\left\|x_{k}\right\|\right)\right]\left\|x_{k}\right\| d t\right) g\left(\rho_{k}\right)^{-1} \\
\leq & \left(\int_{0}^{\rho_{k}} w(s) d s+\int_{0}^{\rho_{k}} e\left(\rho_{k}\right) d s+c \rho_{k}\right) g\left(\rho_{k}\right)^{-1}=\rho_{k+1},
\end{aligned}
$$

since

$$
x_{k} \in U\left(0,\left\|x_{k}\right\|\right) \subseteq U\left(0, \rho_{k}\right)
$$

and

$$
\begin{aligned}
\| \int_{0}^{1} A_{k}(0)^{-1}\left(\left(f_{1 k}^{\prime}\left(t x_{k}\right)-A_{k}\left(x_{k}\right)\right) x_{k} d t \|\right. & \leq \int_{0}^{1}\left(w\left(t\left\|x_{k}\right\|\right)+c\right)\left\|x_{k}\right\| d t \\
\| A_{k}(0)^{-1}\left(f_{2 k}\left(x_{k}-f_{2 k}(0)\right) \|\right. & \leq e\left(\left\|x_{k}\right\|\right)\left\|x_{k}\right\|
\end{aligned}
$$

by (4) and (5) respectively.

Hence (9) holds for $k+1$. From relation (14) we conclude that $x_{k+1} \in$ $U(0, R)$. Finally, by letting $k \rightarrow \infty$ in (14) we get $x_{k} \rightarrow 0$, which completes the proof.

In practical cases we can select $A_{k}\left(x_{k}\right)$ to be either $f_{1 k}^{\prime}\left(x_{k}\right)$ or $f_{1 k}^{\prime}\left(x_{0}\right)$ or $f_{1 k}^{\prime}(0)$ or $S_{k}\left(x_{k-1}, x_{k}\right)$ (secant mappings) or any other linear mapping satisfying relations (3)-(5).

Results for the above special methods can now easily follow.

\section{Rèferences}

[1] I.K. Argyros, "Newton-like methods under mild differentiability conditions with error analysis". Bull. Austral. Math. Soc. 37,2, (1987), 131-147. 
[2] J.E. Dennis, "Toward a unified convergence theory for Newton-like methods". In: Nonlinear functional analysis and applications (L. B. Rall, ed.), 425-472, Academic Press, New York, 1971.

[3] T. Fujimoto, "Global asymptotic stability of nonlinear difference equations", I. Econ. Letters, 22, (1987), 247-250.

[4] L.V. Kantorovich, \& G.P. Akilov, "Functional analysis in normed spaces". Pergamon Press, New York, 1964.

[5] J.P. LaSalle, "The stability and control of discrete processes". Springer-Verlag, New York, 1986.

[6] K. Okuguchi, "Mathematical foundation in economical analysis". McGraw-Hill, Tokyo, 1977 (in Japanese).

[7] K. Okuguchi, \& F. Szidarovszky, "Theory of oligopoly with multi-product firms". Springer -Verlag, New York, 1990.

[8] J.M. Ortega, \& W.C. Rheinboldt, "Iterative solutions of nonlinear equations in several variables". Academic Press, New York, 1970.

[9] E. Polak, "Computational methods in optimization: A unified approach". Academic Press, New York, 1971.

[10] W.C. Rheinboldt, "A unified convergence theory for a class of iterative processes". SIAM J. Num. Anal. 5,(1968), 42-63.

[11] S. Tishyadhigama, E. Polak, \& R. Klessig, "A comparative study of several convergence conditions for algorithms modeled by point-to-set maps." Mathematical Programming Study, 10, (1979), 172-190.

[12] T. Yamamoto, \& X. Chen, "Convergence domains of certain iterative methods for solving nonlinear equations". Numer. Funct. Anal. \& Optimiz. 10, (1 \& 2), (1989), 34-48.

[13] P.P Zabrejko, \& D.F. Nguen, "The majorant method in the theory of Newton-Kantorovich approximations and the Ptak-error estimates". Numer. Funct. Anal. \& Optimiz. 9, (1987), 671-684.

Department of Mathematics, Cameron University, Lawton, Ok 73505, U.S.A.

Department of Systems and Industrial Engineering, University of Arizona, Tucson, AZ 85721, U.S.A. 